\title{
MiR-129-5p Sensitizes the Response of Her-2 Positive Breast Cancer to Trastuzumab by Reducing Rps 6
}

\author{
Xiangdong $\mathrm{Lu}^{\mathrm{a}}$ Jingjing Mac Jiahui Chu ${ }^{\mathrm{d}}$ Qing Shao ${ }^{\mathrm{a}}$ Yao Zhang ${ }^{\mathrm{a}}$ \\ Guangping $\mathrm{Lu}^{\mathrm{d}}$ Jun Lid ${ }^{\mathrm{d}}$ Xiang Huang ${ }^{\mathrm{d}}$ Wei Lid ${ }^{\mathrm{d}}$ Yongfei Lid \\ Yang Ling ${ }^{\mathrm{b}}$ Tao Zhao ${ }^{\mathrm{a}, \mathrm{b}}$
}

aDepartment of Oncolgy, Jiangyin People's Hospital Affiliated to Southeast University, Jiangyin, 'Department of Oncolgy, Changzhou tumor hospital Affiliated to Suzhou University, Changzhou, 'Department of Breast, Obstetrics and Gynecology Hospital Affiliated to Nanjing Medical University, Nanjing, 'Department of Oncolgy, The First Affiliated Hospital of Nanjing Medical University, Nanjing, China

\section{Key Words}

Her-2-positive breast cancer $\bullet$ Trastuzumab resistance $・$ MiR-129-5p $\bullet$ RpS6

\begin{abstract}
Background/Aims: Trastuzumab is an important treatment used for patients with Her2-positive breast cancer, but an increasing incidence of trastuzumab resistance has been observed clinically during the past decade. Aberrant microRNA (miR) expression levels are correlated with prognosis and response to trastuzumab in breast cancer. MiR-129-5p is downregulated in trastuzumab-resistant human breast cancer cells (JIMT-1), but its potential function and underlying mechanism remain unclear. Methods: Quantitative RT-PCR (qRT-PCR) was used to determine the expression levels of miR-129-5p and its potential target genes. The effects of miR-129-5p on cell responses to trastuzumab were analyzed by CCK- 8 and flow cytometry assays in Her-2-positive breast cancer cells (SKBR-3 and JIMT-1). Bio-informatics analyses were performed to predict target genes of miR-129-5p, and luciferase assays were carried out to confirm the binding of miR-129-5p and rpS6. Results: MiR-129-5p, which was downregulated and predicted to target rpS6 in trastuzumab-resistant breast cancer cells, enhanced the sensitivity of breast cancer cells to trastuzumab by reducing the expression of rpS6. Moreover, the overexpression of rpS6 reversed the sensitivity of cells to trastuzumab induced by miR-129-5p. Conclusions: MiR-129-5p sensitized Her-2-positive breast cancer to trastuzumab by downregulating rpS6. These findings provide novel insights into the common role of rpS6 and its related molecular mechanisms in mediating trastuzumab-resistance in Her-2-positive breast cancers.

X. Lu, J. Ma and J. Chu contributed equally to this work.

Tao Zhao

and Yang Ling
Department of Oncolgy, Jiangyin People's Hospital Affiliated to Southeast University, 163 Shoushan Rd, Jiangyin (China)

Tel. +860510 86879066, E-Mail taoz_soochow@sina.com, medilyn@vip.126.com

\section{KARGER}




\section{Cellular Physiology Cell Physiol Biochem 2017;44:2346-2356

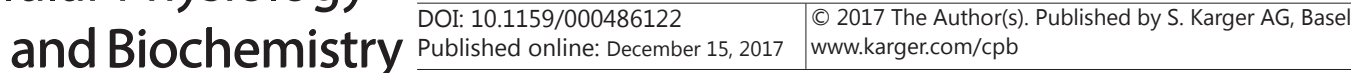

Lu et al.: miR-129-5p Sensitize Breast Cancer to Trastuzumab via Rps6

\section{Introduction}

ErbB-2/Her-2 is amplified in $20-30 \%$ of human invasive breast cancers and is associated with a poor prognosis and reduced disease-free survival rates $[1,2]$. The humanized antiHer-2 antibody trastuzumab (Herceptin) has significantly increased response rates in Her-2positive breast cancer patients; however, most patients who respond to the initial treatment develop resistance within 1 year [3]. Furthermore, trastuzumab-resistant breast cancers have been identified to be more aggressive [4]. Hence, it is necessary to clarify the underlying mechanisms of trastuzumab resistance to develop new strategies for treating Her-2-positive breast cancer.

During the past decades, it has been confirmed that approximately 70\% of Her-2-positive breast cancers receive few benefits from trastuzumab treatment [3], which indicates that Her-2 expression is not the only factor involved in the response of cancers to trastuzumab. A series of studies found that aberrant PI3K/Akt/mTOR/rpS6 signaling activity and loss of PTEN expression contributed greatly to trastuzumab resistance in breast cancers [5-7]. Recently, many miRNAs, such as miR-210, miR-375, and miR-200c, have been found play important roles not only in chemoresistance but also in trastuzumab resistance [8-10].

MiRNAs belong to a class of small non-coding RNA molecules that are 19-25 nucleotides in length; they regulate the expression of a variety of genes via sequence-specific binding to the 3'-untranslated region (3'-UTR) of target mRNAs, which results in the degradation of target mRNAs or inhibition of the mRNA-protein translation process [11, 12]. MiRNAs are phylogenetically conserved, and over $50 \%$ of miRNAs are located in cancer-associated genomic regions [13], which indicates that miRNAs may play important roles in a variety of biological processes [14], such as tumorigenesis. Additionally, miRNAs are also closely associated with drug resistance. Jung et al. found that miR-210, a hypoxia-induced miRNA in MCF-7 breast cancer cells, was significantly upregulated in trastuzumab-resistant BT474 cells [8]. Hypoxia is an important initiator of chemoresistance in cancers; therefore, miR-210 might mediate hypoxia-induced chemoresistance in breast cancer. Additionally, the authors confirmed that circulating miR-210 levels are associated with trastuzumab sensitivity. In another study, Bai et al. demonstrated that miR-200c, a well-known chemoresistancerelated miRNA, could induce trastuzumab resistance by regulating epithelial-mesenchymal transition (EMT) in breast cancer cells [10] hough chemoresistance-related miRNAs have been recognized as regulatory molecules in trastuzumab-resistant breast cancers, their underlying mechanisms remain unclear.

MiR-129-5p, which is downregulated in breast cancer, has been related to EMTmediated chemoresistance and poor prognosis in breast cancer [15]. Interestingly, rpS6, a post-treatment indicator of Her-2 signaling-induced drug resistance, is a downstream target of not only mTOR $[16,17]$ but also miR-129-5p. Hence, we propose that miR-129-5p might inhibit trastuzumab resistance through downregulating rpS6 in Her-2-positive breast cancer cells. In this study, we aimed to identify the effect of miR-129-5p on trastuzumab sensitivity and elucidate the underlying mechanism.

\section{Materials and Methods}

\section{Patients and samples}

Patients were identified through electronic records obtained from Jiangyin Peoples' Hospital. The study was approved by the Ethics Committee of the Jiangyin Peoples' Hospital. Blood samples were collected from 32 women with Her-2 amplified breast cancer after obtaining informed consent between 2012 and 2014. The status of Her-2 was detected by immunohistochemistry. The specimens with $2+$ immunostaining scores were identified as HER2 amplification if the Her-2 (red arrow): CEP17 (green arrow) ratio was greater than or equal to 2 by fluorescence in situ hybridization (Fig. 1). All the patients were treated with trastuzumab during the adjuvant and/or advanced treatment. Trastuzumab primary resistance was defined as progression at first radiological reassessment at 8-12 weeks or within 3 months after first-line 


\section{Cellular Physiology Cell Physiol Biochem 2017;44:2346-2356 \begin{tabular}{l|l|l}
\hline DOI: 10.1159/000486122 & $\begin{array}{l}\text { () 2017 The Author(s). Published by S. Karger AG, Basel } \\
\text { www.karger.com/cpb }\end{array}$
\end{tabular}}

Lu et al.: miR-129-5p Sensitize Breast Cancer to Trastuzumab via Rps6

trastuzumab in the metastatic setting or new recurrences diagnosed during or within 12 months after adjuvant trastuzumab [18]. Whole blood samples were collected in EDTAcontaining lavender top tubes (Becton Dickinson, NJ) before the treatment of trastuzumab and chemotherapy. Then the samples were centrifuged at 3, $000 \mathrm{~g}$ for 10 minutes, and the serum supernatant was transferred to new tubes for subsequently experiments.

\section{Cell culture}

Human breast cancer cell lines SKBR3 (trastuzumab-sensitive) and JIMT-1 (trastuzumab-resistant) were obtained from the American Type Culture Collection (ATCC, Manassas, VA, USA). SKBR3 cells were cultured

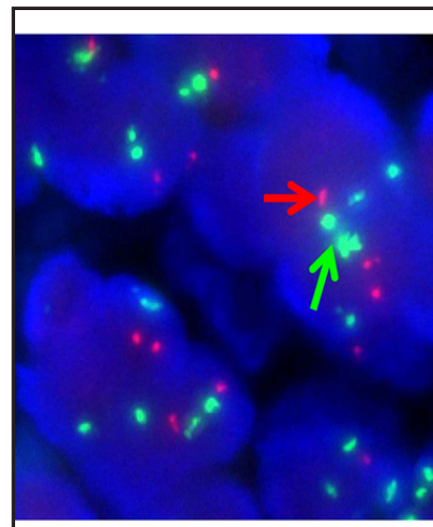

Her-2 negative

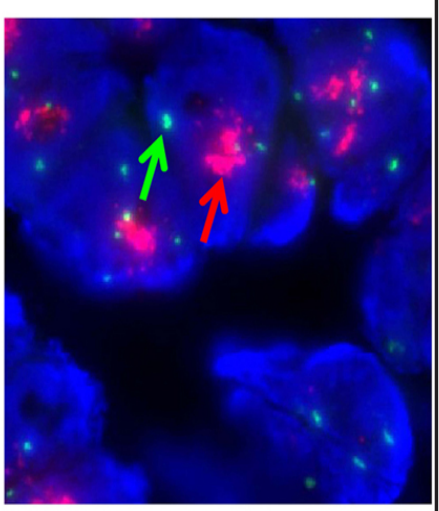

Her-2 positive

Fig. 1. Identification of Her-2-positive breast cancer samples by FISH. The specimens with 2+ immunostaining scores were identified as Her-2-positive if the Her-2 (red arrow): CEP17 (green arrow) ratio was greater than or equal to 2 according to fluorescence in situ hybridization. in RPMI-1640 (Multicell, USA), and JIMT-1cells were cultured in DMEM (Gibco, UK) with 10\% Fetal Bovine Serum (FBS, Gibco), containing $100 \mathrm{U} / \mathrm{ml}$ penicillin and $100 \mu \mathrm{g} / \mathrm{ml}$ streptomycin (Multicell, USA). Cells were cultured at humidified $37^{\circ} \mathrm{C}$ in $5 \% \mathrm{CO}_{2}$ and the cell medium was replaced every $48 \mathrm{~h}$.

\section{Methyl thiazolyl tetrazolium (MTT) assay}

SKBR3 and JIMT-1 cells were seeded in 96-well plates at a density of 4000 cells per well and incubated for $24 \mathrm{~h}$. After incubation with different doses of trastuzumab (0, 2, 20, 200, $2000 \mu \mathrm{g} / \mathrm{ml})$ for $24 \mathrm{~h}, 48 \mathrm{~h}$ and $96 \mathrm{~h}$ at $37^{\circ} \mathrm{C}, 20 \mathrm{uL}$ of 3-(4, 5-di methylthiazol-2-yl)-2, 5-diphenyltetrazoliumbromide (MTT, Sigma Aldrich) solution ( $5 \mathrm{mg} / \mathrm{mL}$ in phosphate buffered saline) was added to each well, and the incubation was continued for an additional $4 \mathrm{~h}$ at $37^{\circ} \mathrm{C}$. Then, the medium was rapidly removed, and the MTT crystals were solubilized in $150 \mathrm{~mL}$ DMSO. The absorbance of each well was read in a plate reader at $490 \mathrm{~nm}$ [19]. The absorbance readings were subtracted from the value of the blank wells; the reduction in cell growth was calculated as percentage of the control absorbance in the absence of any drug. The data represent the mean \pm SD of at least three independent experiments.

\section{Target prediction and functional annotations of miR-129-5p}

The potential targets of miRNAs were predicted using the Target-Scan, PicTar, and microRNA.org programs with the default parameters [20]. Intersecting elements identified in each miRNA target prediction program were manually selected as miRNA targets. To investigate the possible biological processes regulated by the miRNAs, the MAS3 program (http://www.bioinfo.capitalbio.com/mas3/) was used to determine the putative functions of the potential miRNA target genes by annotation using the Gene Ontology (GO) and Kyoto Encyclopedia of Genes and Genomes (KEGG) analyses.

Quantification Real-time RT-PCR (qRT-PCR)

Total RNA of samples was isolated using Trizol reagent (Invitrogen) according to the manufacturer's protocol [21]. The purified total RNA $(1 \mu \mathrm{g})$ was then reverse transcribed using the PrimeScript RT reagent kit (Takara, Japan), and qPCR was performed on an ABI 7900 PCR System (Applied Biosystems, USA) using Power SYBR Green PCR Master Mix (2X, Applied Biosystems). The primers are: miR-129-5p (F) 5'-GGGGGCTTTTTGCGGTCTGG-3', miR-129-5p (R) 5'-AGT GCGTGTCGTGGAGTC-3'; U6 (F) 5' -CTCGCTTCGGCAGCACA-3, U6 (R) 5'- AACGCTTCACGAATTTGCGT-3'; rpS6 (R) 5'-GTCATCCAGCATGGGTGCTG-3' and rpS6 (F) 5'-GGCTGATACCTTTTGGGACAG-3'; GAPDH (F) 5'-CGCGCCC CCGG TTTCTA-3', GAPDH (R) 5'-[22]GGCTCGGCTGGCGAC-3'. Each reaction was performed in triplicate. The relative expression levels were determined using the comparative threshold cycle $2^{\wedge \wedge} \mathrm{Ct}$ analysis method. 
Lu et al.: miR-129-5p Sensitize Breast Cancer to Trastuzumab via Rps6

\section{Western blotting}

After the cell protein components were collected, a standard western blotting analysis was used to investigate the rpS6 expression levels. Briefly, Protein lysates were boiled in SDS-sample buffer for 5 min and then subjected to 8\% SDS polyacrylamide gel electrophoresis and transferred to PVDF (polyvinylidene fluoride) membranes (Bio-Rad). Membranes were then blocked for $2 \mathrm{~h}$ in $5 \%$ milk-Tris-Buffered Saline Tween-20 (TBST) at room temperature, and incubated overnight at $4{ }^{\circ} \mathrm{C}$ either with the monoclonal antibodies (Cell Signaling Technology, USA) of anti-GAPDH, or anti-rpS6. Membranes were washed four times in TBST and incubated with appropriate horseradish peroxidase-conjugated secondary antibodies at room temperature for $2 \mathrm{~h}$. Blots were visualized by enhanced chemiluminescence (Thermo, USA) and analyzed using a scanning densitometer with the molecular analysis software FluorChem M system (Protein Simple, USA).

\section{Luciferase Reporter Assays}

The 3'-UTR of the wild-type rpS6 and a variant containing mutations in the putative miR-129-5p binding site (Fig. 6A) were inserted downstream of the firefly luciferase gene in the pGL3 vector (Promega, Madison, USA). Trastuzumab-resistant JIMT-1 cells were co-transfected with reporter constructs, an internal control vector (pGL4.73), and synthetic miR-129-5p mimics. $48 \mathrm{~h}$ after transfection, cells were rinsed with phosphate buffered saline (PBS), and then luciferase activity was assayed using the Dual-Luciferase Reporter Assay System (Promega, USA) and a luminometer [23]. The luciferase activity of each lysate was normalized to the activity of Renilla luciferase driven by the constitutively expressing promoter in the phRL vector. Basal promoter activity was measured as the fold change relative to the activity observed with the basic pGL3 vector alone.

\section{Statistical analysis}

The differences between the treated and the control cells about protein expressions were by applying one way ANOVA (analysis of variance) method followed by standard student t-test analysis. A p-value of less than 0.05 was considered statistically significant, each experiment was performed at least triple times. Computer-based calculations were conducted using SPSS version 20.0 (SPSS Inc. Chicago, Illinois, USA).

\section{Results}

\section{Resistance index of SKBR-3 and JIMT-1 cells for trastuzumab}

To assess the resistance index of SKBR-3 and JIMT-1 cells for the anti-Her-2 agent trastuzumab, cell viability was determined by MTT assays. SKBR-3 and JIMT-1 cells were treated with different concentrations of trastuzumab $(0,2,20,200$, or $2000 \mu \mathrm{g} / \mathrm{ml})$. The results showed that the IC50 for SKBR-3 cells was 63.25 $\mu \mathrm{g} / \mathrm{ml}$ trastuzumab. However, the IC50 for JIMT-1 cells was $169.6 \mu \mathrm{g} / \mathrm{ml}$ trastuzumab; this IC50 was increased 2.68fold compared with the IC50 of SKBR-3 cells (Fig. 2). These results are consistent with those of a previous report indicating that JIMT-1 cells are resistant to trastuzumab [24].

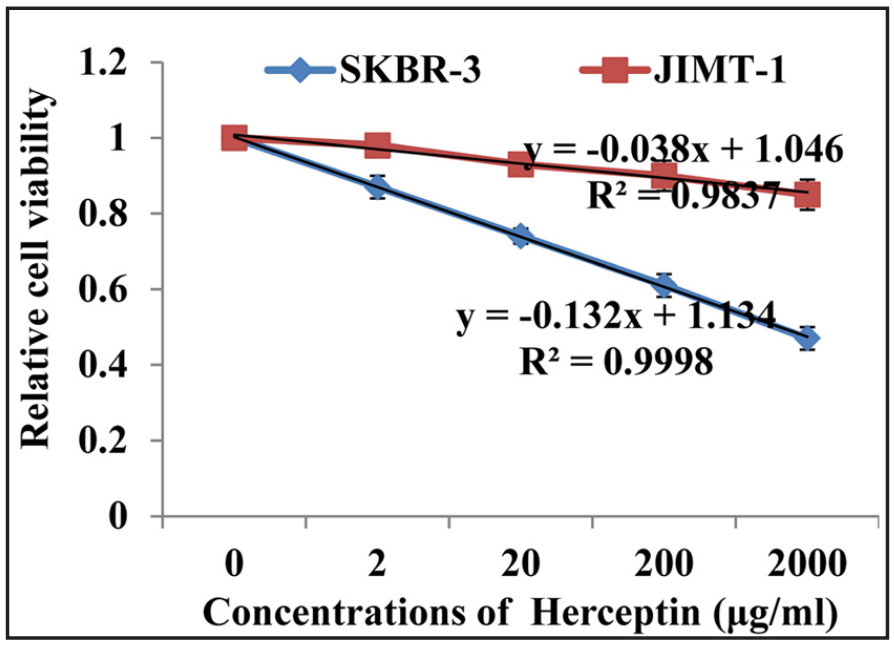

Fig. 2. Resistance index of SKBR-3 and JIMT-1 cells for trastuzumab. The data show that the resistance index of JIMT-1 cells was 2.68-fold higher than that of SKBR-3 cells. 


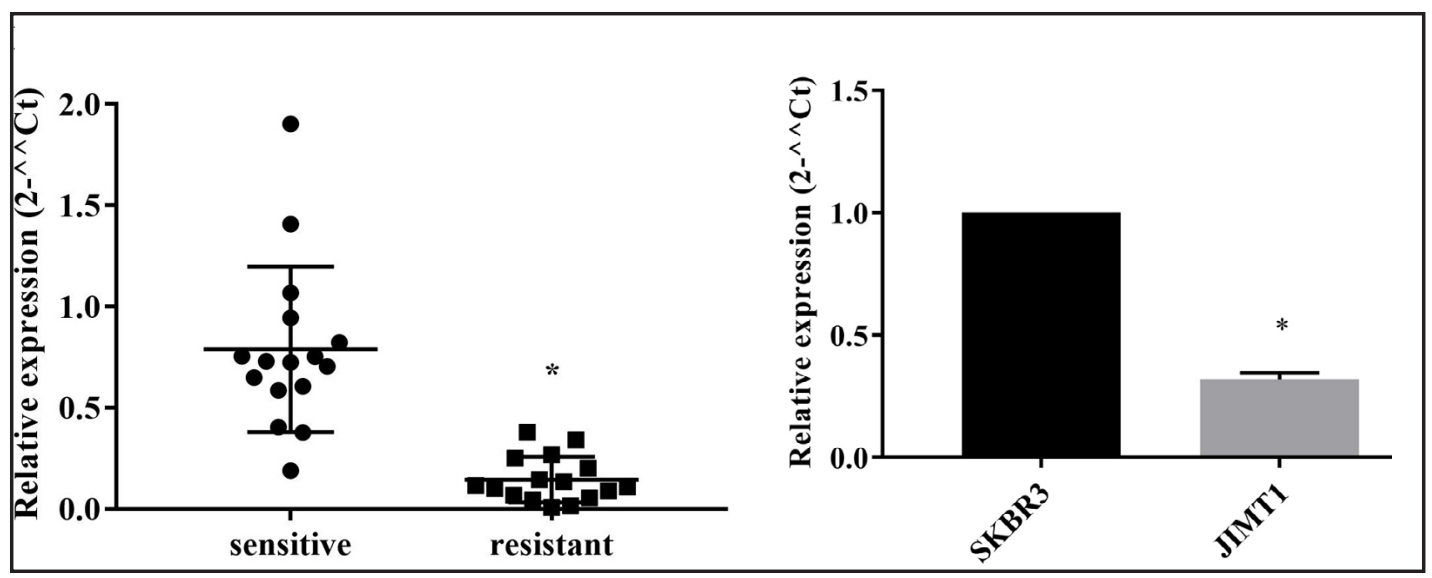

Fig. 3. Expression pattern of miR-129-5p in trastuzumab-resistant breast cancers. miR-129-5p levels were significantly decreased in trastuzumab-resistant serum (A) and cell (B) samples.

\section{Expression pattern of miR-129-5p in trastuzumab-resistant breast cancers}

It has been reported that low levels of miR-129-5p are correlated with breast cancer chemoresistance. In this study, the expression levels of miR-129-5p were measured in the serum of Her-2-positive breast cancer patients. The data showed that miR-129-5p levels were significantly lower in trastuzumab-resistant samples than trastuzumab-sensitive samples (Fig. 3A). Similar to the blood sample results, miR-129-5p was also downregulated in trastuzumab-resistant JIMT-1 cells compared to trastuzumab-sensitive SKBR-3 cells (Fig. 3B).

MiR-129-5p increased trastuzumab sensitivity in Her-2-positive breast cancer cells

To identify the effects of miR-129-5p on the sensitivity of breast cancer cells to trastuzumab, JIMT-1 cells in which miR-129-5p mimics were used to overexpress miR-129$5 p$ were used. After transfection, miR-129-5p levels were increased approximately 3.21-fold compared to those of the scrambled mimic control group (Fig. 4A). Moreover, cell viability was low in the mimic group treated with trastuzumab (Fig. 4B), which indicated that miR129-5p overexpression increased trastuzumab sensitivity in JIMT-1 cells. In contrast, SKBR3 cells in which miR-129-5p was overexpressed were transfected with the miR-129-5p sponge. After transfection, miR-129-5p levels were decreased 2.17-fold compared to those in the scrambled mimic control group (Fig. 4C). Cell viability was high in the miR-129-5p sponge group treated with trastuzumab (Fig. 4D), which indicated that downregulating miR129-5p decreased trastuzumab sensitivity in SKBR-3 cells.

\section{GO and pathway analyses of miR-129-5p target genes}

We predicted the potential targets and function of miR-129-5p by using bio-informatics tools. GO analysis results showed that genes in the biological processes of the positive regulation of RNA polymerase II promoter, negative regulation of RNA polymerase II promoter transcription, DNA-templated transcription from the RNA polymerase II promoter, positive regulation of transcription, DNA-templated regulation of transcription, DNA-templated negative regulation of transcription, DNA-templated axon guidance, positive regulation of the nuclear-transcribed mRNA catabolic process, and adult behavior were enriched (Fig. 5A). The molecular functions of these genes include protein binding, DNA binding, transcription factor activity, and sequence-specific DNA binding (Fig. 5B). These genes are primarily located in the nucleus, nucleoplasm, cytoplasm, and intracellular ribonucleoprotein cytoplasm (Fig. 5C). Then, we used pathway mapping tools to identify the biological pathways of these genes; 10 categories were found, including the calcium signaling pathway, stem cell pluripotency regulation, the oxytocin signaling pathway, adrenergic signaling in cardiomyocytes, the TGFbeta signaling pathway, and the cAMP signaling pathway (Fig. 5D). 


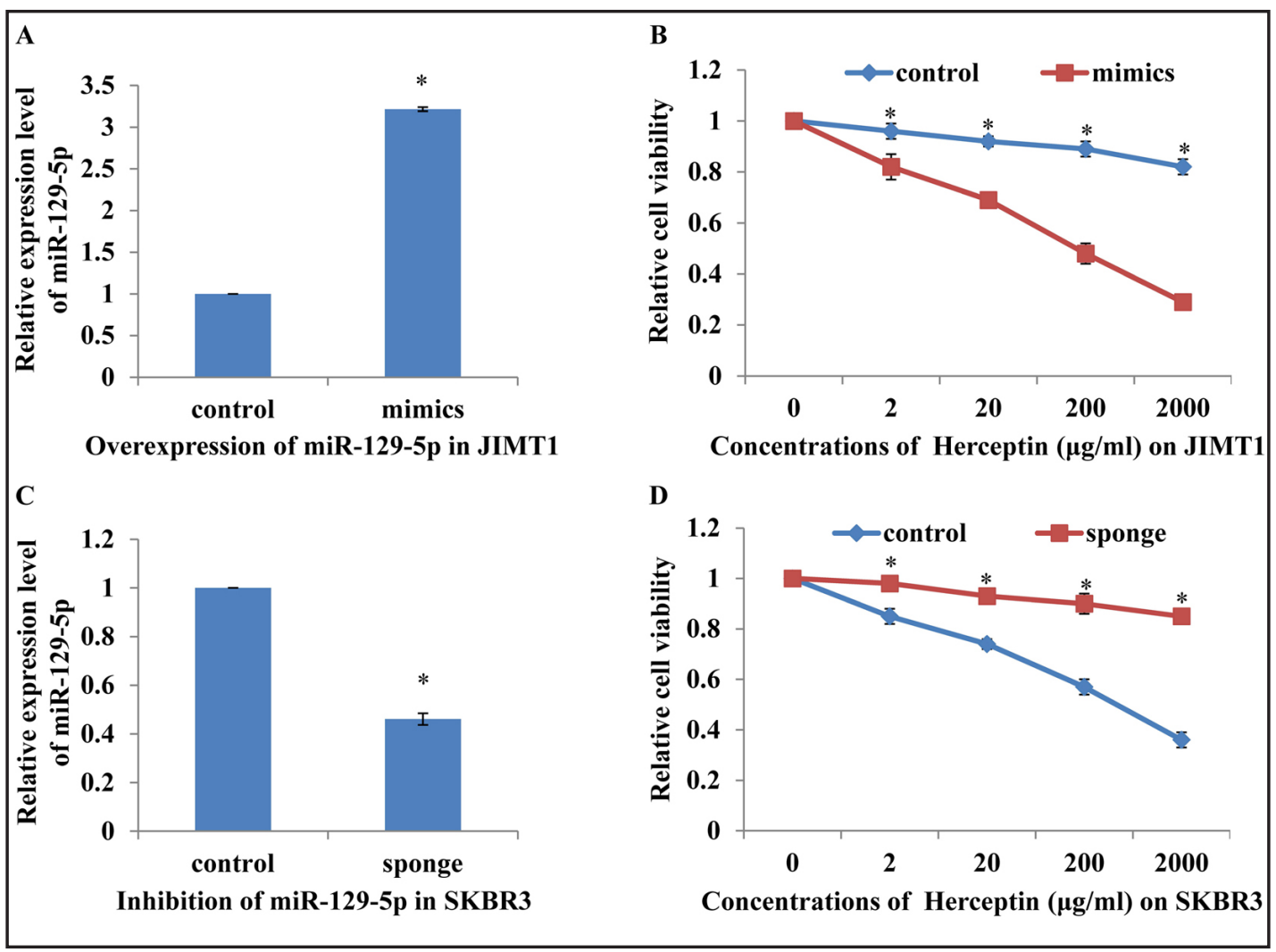

Fig. 4. MiR-129-5p increased trastuzumab sensitivity in Her-2-positive breast cancer cells. MiR-129-5p overexpression increased the sensitivity of JIMT-1 cells to trastuzumab (A \& B). In contrast, miR-129-5p knockdown reduced the sensitivity of SKBR-3 cells to trastuzumab (C \& D).

MiR-129-5p reduced the expression levels of the rpS6 gene by targeting the 3'-UTR region of rpS6 $m R N A$

To confirm the miR-129-5p binding sites within the 3'-UTR region of the rpS6 gene, we constructed a wild type (wt) and mutant (mut) miR-129-5p binding sites in the rpS6 3'-UTR (Fig. 6A). Then, we evaluated the effects of the mutant and wild type constructs in miR-1295 p-overexpressing JIMT-1 cells (mimic group) using a dual-luciferase analysis system. The results showed that the luciferase activity was significantly lower in the mimic group cotransfected with the wild type 3'-UTR construct than the mimic group co-transfected with the mutant construct (Fig. 6B), suggesting that miR-129-5p suppressed rpS6 gene expression by binding to the 3'-UTR of rpS6 mRNA. Consistent with the luciferase analysis data, miR-129$5 p$ overexpression decreased the mRNA and protein expression levels of rpS6 compared to those in the scrambled mimic groups, whereas miR-129-5p inhibition increased the mRNA and protein expression levels of rpS6 compared to those in the scrambled mimic groups (Fig. $6 \mathrm{C} \& 6 \mathrm{D})$. These results indicate that miR-129-5p reduced the expression levels of the rpS6 gene by targeting the 3'-UTR region of rpS6 mRNA in breast cancer cells.

MiR-129-5p regulated trastuzumab sensitivity in Her-2-positive breast cancer cells through rpS6

A rescue strategy was adopted to confirm the function of rpS6 in miR-129-5p induced trastuzumab-sensitivity. SKBR-3 cells were co-transfected with the miR-129-5p mimic/ rpS6 vector or mimic/empty vector (pcDNA-3.1-rpS6 or pcDNA-3.1); JIMT-1 cells were cotransfected with the miR-129-5p sponge/rpS6-siRNA or sponge/scrambled control. The expression levels of rpS6 were determined by qRT-PCR and western blotting (Fig. 7A \& 7B). Moreover, after rpS6-siRNA treatment, the trastuzumab resistance induced by miR-129-5p 
Lu et al.: miR-129-5p Sensitize Breast Cancer to Trastuzumab via Rps6

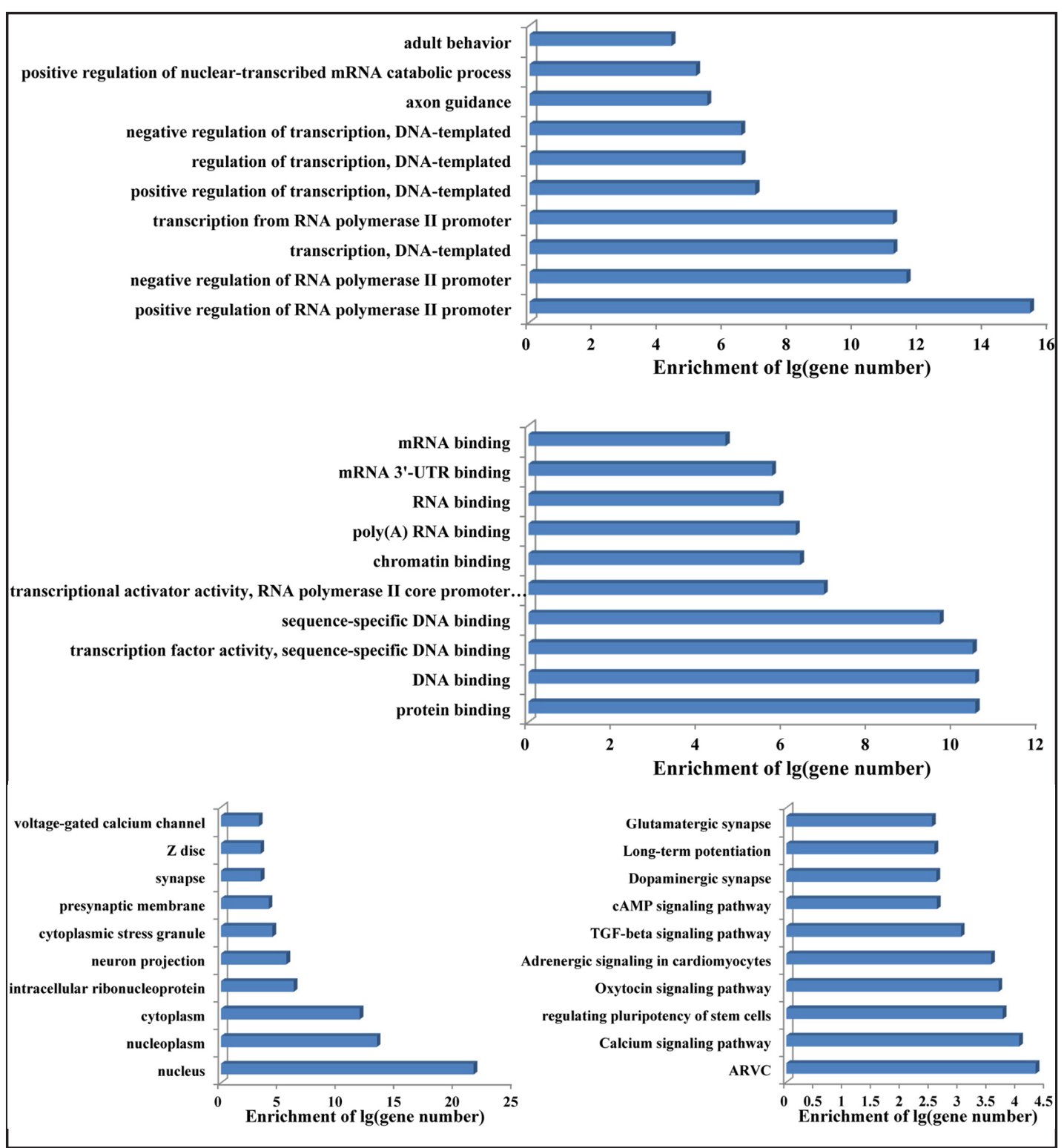

Fig. 5. GO and pathway analyses of miR-129-5p target genes. GO analysis results showed that genes involved in the biological processes of the positive regulation of RNA polymerase II promoter, negative regulation of RNA polymerase II promoter transcription, and DNA-templated transcription from the RNA polymerase II promoter were enriched (A). The molecular functions of these genes included protein binding and DNA binding (B), and these genes are located primarily in the nucleus, nucleoplasm, and cytoplasm (C). KEGG pathway analyses indicated that these genes are involved in the calcium signaling pathway, stem cells pluripotency regulation, the oxytocin signaling pathway, adrenergic signaling in cardiomyocytes, the TGF-beta signaling pathway, and the cAMP signaling pathway (D).

inhibition was significantly reduced in SKBR-3 cells; in contrast, the trastuzumab sensitivity induced by miR-129-5p overexpression was reversed by rpS6 overexpression in JIMT-1 cells (Fig. 7C \& 7D). These results demonstrated that miR-129-5p could induce trastuzumab sensitivity in Her-2-positive breast cancer cells through regulating rpS6 expression levels. 


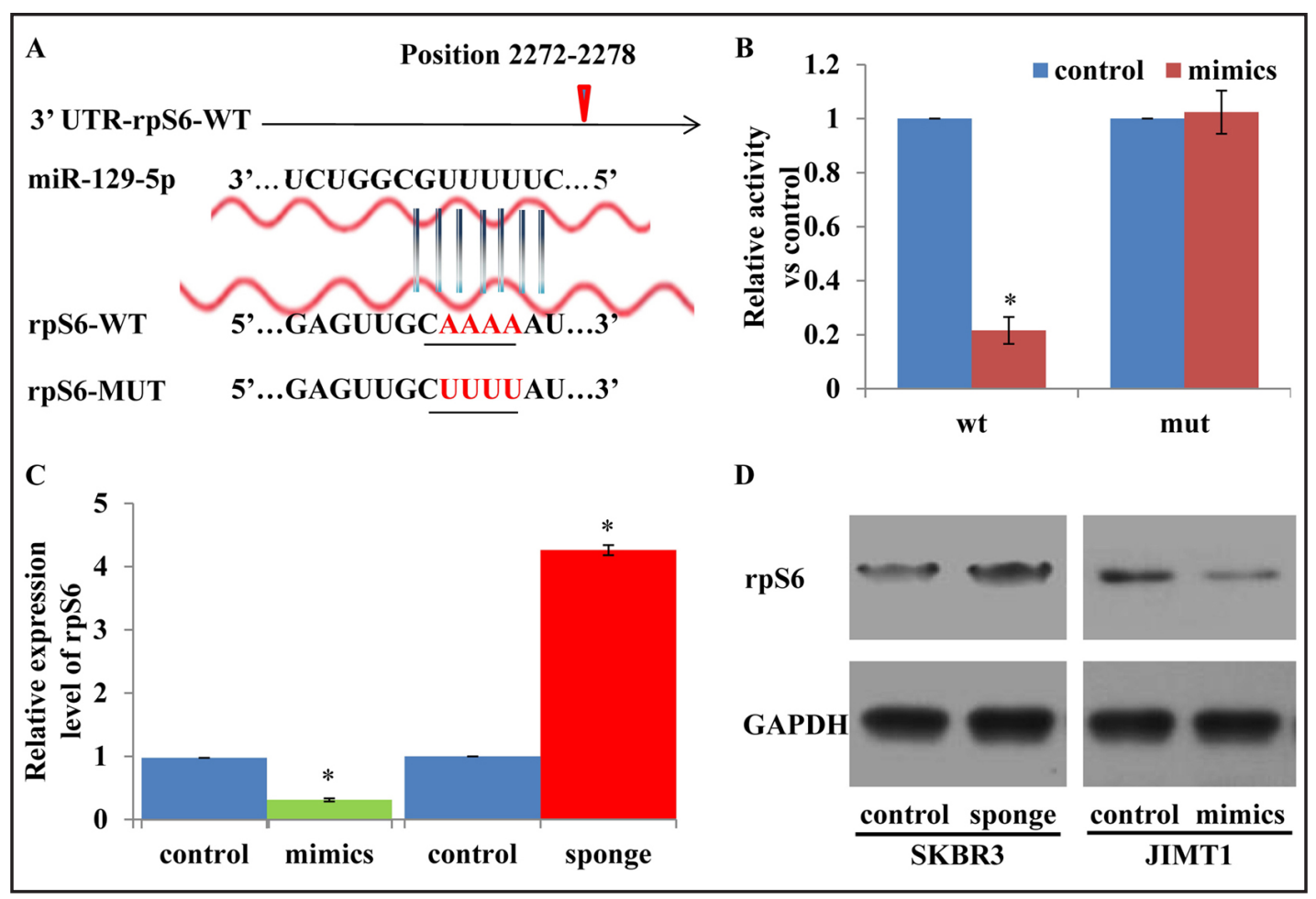

Fig. 6. MiR-129-5p reduced the expression levels of the rpS6 gene by targeting the 3'-UTR region of rpS6 mRNA. We constructed wild type (wt) and mutant (mut) miR-129-5p binding sites in the rpS6 3'-UTR (A). The luciferase activity was significantly lower in the mimic group co-transfected with the wild type 3'-UTR construct than the mimic group co-transfected with the mutant construct (B). MiR-129-5p overexpression decreased the mRNA and protein expression levels of rpS6 compared to those of the scrambled groups, whereas miR-129-5p inhibition increased the mRNA (C) and protein expression (D) levels of rpS6 compared to those of the scrambled groups.

\section{Discussion}

Over the past decade, trastuzumab has been used successfully for the clinical treatment of patients with Her-2-positive breast cancers. However, trastuzumab resistance has become the major obstacle to achieving positive clinical outcomes. Her-2 gene amplification is necessary, but not sufficient, for responsiveness to trastuzumab. It has been confirmed that aberrant PI3K/Akt/mTOR/rpS6 signaling activity contributes to acquired resistance to trastuzumab in breast cancers [6]. Recently, many chemoresistance-related miRNAs have been found to regulate the sensitivity of breast cancer cells to trastuzumab. MiR-129-5p has been correlated with EMT-mediated chemoresistance and poor prognosis in breast cancer. Interestingly, rpS6, a downstream target of the mTOR signaling pathway, was predicted to be a target gene of miR-129-5p. In this study, we proposed that downregulating miR-129$5 p$ contributed to trastuzumab resistance in Her-2-positive breast cancer cells through increased rpS6 expression.

The identification of patients who might benefit from chemotherapy is important because the individualized selection of therapeutic agents could maximize the benefits and minimize the side-effects of ineffective therapies [25]. MiRNA expression analyses are effective tools for predicting therapeutic responses to clinical treatments, especially when used in conjunction with well-established gene expression array-based profiling technology. MiRNAs can play differing roles in regulating cancer occurrence and drug resistance by targeting oncogenes or tumor suppressors. Dysregulated expression levels of miRNAs are correlated with trastuzumab resistance in breast cancers [8-10] In this study, we found

\section{KARGER}




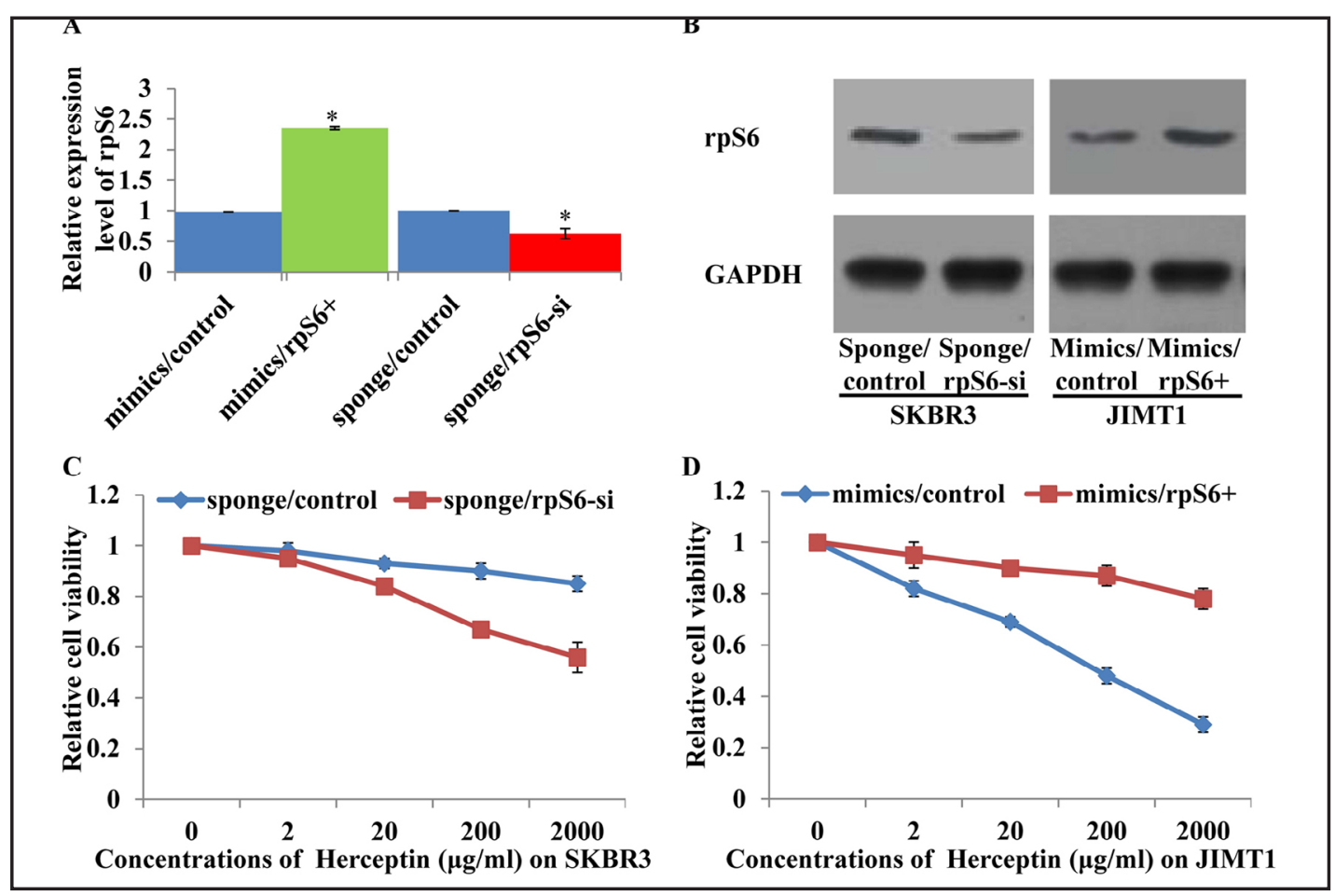

Fig. 7. MiR-129-5p regulated trastuzumab sensitivity in Her-2-positive breast cancer cells through rpS6. SKBR-3 cells were co-transfected with the miR-129-5p mimic/rpS6 vector or mimic/empty vector; JIMT1 cells were co-transfected with the miR-129-5p sponge/rpS6-siRNA or sponge/scrambled control. The expression levels of rpS6 were determined by qRT-PCR (A) and western blotting (B). After rpS6-siRNA treatment, the miR-129-5p-induced inhibition of trastuzumab resistance was significantly suppressed in SKBR-3 cells (C); in contrast, trastuzumab sensitivity induced by miR-129-5p overexpression was reversed by rpS6 overexpression in JIMT-1 cells (D).

that miR-129-5p was downregulated in trastuzumab-resistant serum samples and cells (Fig. 3), which is consistent with the expression patterns found in chemoresistant samples. Furthermore, the upregulation of miR-129-5p increased the sensitivity of Her-2-positive breast cancer cells to trastuzumab (Fig. 4). However, the underlying mechanism is not clear.

RpS6 was predicted to be a target of miR-129-5p by bio-informatics analysis tools. Previous evidence suggests that rpS6 is downstream of the phosphoinositide 3-kinase (PI3K)/Akt/mTOR signaling pathway and is dysregulated in a variety of cancers. Interestingly, rpS6 always participates in regulating translation initiation and thus protein synthesis [26], in accordance with GO term analysis (Fig. 5A); on the other hand, rpS6 phosphorylation level regulates several protein signaling pathways involving TGF- $\beta[27,28]$ accordance with Pathway analysis (Fig. 5D), which suggested that rpS6 probably mediated miR-129$5 p$ induced trastuzumab sensitivity. mTOR exists as 2 distinct complexes, mTOR complex 1 (mTORC1) and mTOR complex 2 (mTORC2), and rpS6 is one of best mTORC2 substrates [29]. Both genetic and biochemical data suggest that the activation of the PI3K/Akt/mTOR/ rpS6 pathway contributes to the overexpression of ER, PR, and Her-2 in breast cancer [30]. In contrast, Her-2-positive tumors can escape the effects of trastuzumab through the PI3K/Akt/ mTOR/rpS6 pathway; combination therapy with mTOR inhibitors could reverse trastuzumab resistance [31, 32]. The data in Fig. 6 show that the altered expression levels of miR-129-5p in Her-2-positive breast cancer cells led to opposite changes in rpS6 expression. Moreover, the rpS6 mRNA 3'-UTR has a miR-129-5p binding site; after miR-129-5p transfection, the expression levels of rpS6 mRNA and protein were both suppressed. However, there were no obvious effects of miR-129-5p on the mutant rpS6 3'-UTR, which highlighted the negative regulation of rpS6 by miR-129-5p.

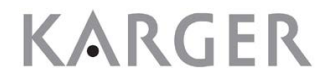




\section{Cellular Physiology Cell Physiol Biochem 2017;44:2346-2356 \begin{tabular}{l|l} 
and Biochemistry Published online: December 15, 2017 & $\begin{array}{l}\text { (c) } 2017 \text { The Author(s). Published by S. Karger AG, Basel } \\
\text { www.karger.com/cpb }\end{array}$ \\
\hline
\end{tabular}}

Lu et al.: miR-129-5p Sensitize Breast Cancer to Trastuzumab via Rps6

To further analyze whether rpS6 functions in miR-129-5p-mediated breast cancer sensitivity to trastuzumab, we performed rescue assays. We found that miR-129-5p increased trastuzumab sensitivity through targeting rpS6 in Her-2-positive breast cancer cells (Fig. 7) because restoring the expression of rpS6 abolished miR-129-5p-mediated breast cancer sensitivity to trastuzumab. In conclusion, we identified that miR-129-5p is suppressed in trastuzumab-resistant breast cancer cells and serum samples. Restoring cellular miR-129$5 p$ levels increased the sensitivity of Her-2-positive breast cancer to trastuzumab by directly targeting rpS6, which is downstream of the PI3K/Akt/mTOR pathway. Our study indicates the therapeutic potential of miR-129-5p in combination with trastuzumab for treating Her2 -positive breast cancers.

\section{Acknowledgements}

This study was financially supported by the Natural Science Foundation of Jiangsu (BL2013012, BE2016656), and Health and Family Planning Commission Foundation of Wuxi (Q201630, Q201601).

\section{Disclosure Statement}

We declare that there is no conflict of interests.

\section{References}

1 Krishnamurti U, Silverman JF: HER2 in breast cancer: a review and update. Adv Anat Pathol 2014;21:100107.

- Pan Z, Jing W, He K, Zhang L, Long X: SATB1 is Correlated with Progression and Metastasis of Breast Cancers: A Meta-Analysis. Cell Physiol Biochem 2016;38:1975-1983.

-3 Jones SE, Collea R, Paul D, Sedlacek S, Favret AM, Gore I Jr, Lindquist DL, Holmes FA, Allison MA, Brooks BD, Portillo RM, Vukelja SJ, Steinberg MS, Stokoe C, Crockett MW, Wang Y, Asmar L, Robert NJ, O’Shaughnessy J: Adjuvant docetaxel and cyclophosphamide plus trastuzumab in patients with HER2-amplified early stage breast cancer: a single-group, open-label, phase 2 study. Lancet Oncol 2013;14:1121-1128.

4 Sahin O, Wang Q Brady SW, Ellis K, Wang H, Chang CC, Zhang Q, Priya P, Zhu R, Wong ST, Landis MD, Muller WJ, Esteva FJ, Chang J, Yu D: Biomarker-guided sequential targeted therapies to overcome therapy resistance in rapidly evolving highly aggressive mammary tumors. Cell Res 2014;24:542-559.

-5 Nahta R, O’Regan RM: Evolving strategies for overcoming resistance to HER2-directed therapy: targeting the PI3K/Akt/mTOR pathway. Clin Breast Cancer 2010;10 Suppl 3:S72-78.

-6 O’Brien NA, McDonald K, Tong L, von Euw E, Kalous O, Conklin D, Hurvitz SA, di Tomaso E, Schnell C, Linnartz R, Finn RS, Hirawat S, Slamon DJ: Targeting PI3K/mTOR overcomes resistance to HER2-targeted therapy independent of feedback activation of AKT. Clin Cancer Res 2014;20:3507-3520.

7 Li G, Shan C, Liu L, Zhou T, Zhou J, Hu X, Chen Y, Cui H, Gao N: Tanshinone IIA inhibits HIF-1alpha and VEGF expression in breast cancer cells via mTOR/p70S6K/RPS6/4E-BP1 signaling pathway. PLoS One 2015;10:e0117440.

8 Jung EJ, Santarpia L, Kim J, Esteva FJ, Moretti E, Buzdar AU, Di Leo A, Le XF, Bast RC Jr, Park ST, Pusztai L, Calin GA: Plasma microRNA 210 levels correlate with sensitivity to trastuzumab and tumor presence in breast cancer patients. Cancer 2012;118:2603-2614.

-9 Ye XM, Zhu HY, Bai WD, Wang T, Wang L, Chen Y, Yang AG, Jia LT: Epigenetic silencing of miR-375 induces trastuzumab resistance in HER2-positive breast cancer by targeting IGF1R. BMC Cancer 2014;14:134.

10 Bai WD, Ye XM, Zhang MY, Zhu HY, Xi WJ, Huang X, Zhao J, Gu B, Zheng GX, Yang AG, Jia LT: MiR-200c suppresses TGF-beta signaling and counteracts trastuzumab resistance and metastasis by targeting ZNF217 and ZEB1 in breast cancer. Int J Cancer 2014;135:1356-1368.

11 Wang QX, Zhu YQ Zhang H, Xiao J: Altered MiRNA expression in gastric cancer: a systematic review and meta-analysis. Cell Physiol Biochem 2015;35:933-944.

12 Lv J, Xia K, Xu P, Sun E, Ma J, Gao S, Zhou Q, Zhang M, Wang F, Chen F, Zhou P, Fu Z, Xie H: miRNA expression patterns in chemoresistant breast cancer tissues. Biomed Pharmacother 2014;68:935-942. 


\section{Cellular Physiology Cell Physiol Biochem 2017;44:2346-2356 \begin{tabular}{l|l} 
and Biochemistry Published 10.1159/000486122 & $\begin{array}{l}\text { (c) } 2017 \text { The Author(s). Published by S. Karger AG, Basel } \\
\text { www.karger.com/cpb }\end{array}$
\end{tabular}}

Lu et al.: miR-129-5p Sensitize Breast Cancer to Trastuzumab via Rps6

13 Zhu X, Fu C, Zhang L, Xu G, Wang S: MiRNAs associated polymorphisms in the 3'UTR of MET promote the risk of non-small cell lung cancer. Cell Physiol Biochem 2015;37:1159-1167.

14 Xie T, Huang M, Wang Y, Wang L, Chen C, Chu X: MicroRNAs as Regulators, Biomarkers and Therapeutic Targets in the Drug Resistance of Colorectal Cancer. Cell Physiol Biochem 2016;40:62-76.

-15 Luan QX, Zhang BG, Li XJ, Guo MY: MiR-129-5p is downregulated in breast cancer cells partly due to promoter $\mathrm{H} 3 \mathrm{~K} 27 \mathrm{~m} 3$ modification and regulates epithelial-mesenchymal transition and multi-drug resistance. Eur Rev Med Pharmacol Sci 2016;20:4257-4265.

16 Meyuhas 0: Ribosomal Protein S6 Phosphorylation: Four Decades of Research. Int Rev Cell Mol Biol 2015;320:41-73.

-17 Esteva FJ, Guo H, Zhang S, Santa-Maria C, Stone S, Lanchbury JS, Sahin AA, Hortobagyi GN, Yu D: PTEN, PIK3CA, p-AKT, and p-p70S6K status: association with trastuzumab response and survival in patients with HER2-positive metastatic breast cancer. Am J Pathol 2010;177:1647-1656.

18 Bartsch R, De Vries C, Pluschnig U, Dubsky P, Bago-Horvath Z, Gampenrieder SP, Rudas M, Mader RM, Rottenfusser A, Wiltschke C, Gnant M, Zielinski CC, Steger GG: Predicting for activity of second-line trastuzumab-based therapy in her2-positive advanced breast cancer. BMC Cancer 2009;9:367.

19 Zhang W, Wang F, Xu P, Miao C, Zeng X, Cui X, Lu C, Xie H, Yin H, Chen F, Ma J, Gao S, Fu Z: Perfluorooctanoic acid stimulates breast cancer cells invasion and up-regulates matrix metalloproteinase-2/-9 expression mediated by activating NF-kappaB. Toxicol Lett 2014;229:118-125.

-20 Lv J, Fu Z, Shi M, Xia K, Ji C, Xu P, Lv M, Pan B, Dai L, Xie H: Systematic analysis of gene expression pattern in has-miR-760 overexpressed resistance of the MCF-7 human breast cancer cell to doxorubicin. Biomed Pharmacother 2015;69:162-169.

21 Lv M, Xu P, Wu Y, Huang L, Li W, Lv S, Wu X, Zeng X, Shen R, Jia X, Yin Y, Gu Y, Yuan H, Xie H, Fu Z: LncRNAs as new biomarkers to differentiate triple negative breast cancer from non-triple negative breast cancer. Oncotarget 2016;7:13047-13059.

22 Zhang S, Ma J, Fu Z, Zhang Z, Cao J, Huang L, Li W, Xu P, Cao X: Promotion of breast cancer cells MDA-MB-231 invasion by di(2-ethylhexyl)phthalate through matrix metalloproteinase-2/-9 overexpression. Environ Sci Pollut Res Int 2016;23:9742-9749.

-23 Wu N, Zhang C, Bai C, Han YP, Li Q: MiR-4782-3p inhibited non-small cell lung cancer growth via USP14 Cell Physiol Biochem 2014;33:457-467.

24 Nam S, Chang HR, Jung HR, Gim Y, Kim NY, Grailhe R, Seo HR, Park HS, Balch C, Lee J, Park I, Jung SY, Jeong KC, Powis G, Liang H, Lee ES, Ro J, Kim YH: A pathway-based approach for identifying biomarkers of tumor progression to trastuzumab-resistant breast cancer. Cancer Lett 2015;356:880-890.

25 Kadia TM, Ravandi F, Cortes J, Kantarjian H: Toward Individualized Therapy in Acute Myeloid Leukemia: A Contemporary Review. JAMA Oncol 2015;1:820-828.

26 Chauvin C, Koka V, Nouschi A, Mieulet V, Hoareau-Aveilla C, Dreazen A, Cagnard N, Carpentier W, Kiss T, Meyuhas O, Pende M: Ribosomal protein S6 kinase activity controls the ribosome biogenesis transcriptional program. Oncogene 2014;33:474-483.

27 Suryawan A, Frank JW, Nguyen HV, Davis TA: Expression of the TGF-beta family of ligands is developmentally regulated in skeletal muscle of neonatal rats. Pediatr Res 2006;59:175-179.

28 Wang CC, Bajikar SS, Jamal L, Atkins KA, Janes KA: A time- and matrix-dependent TGFBR3-JUND-KRT5 regulatory circuit in single breast epithelial cells and basal-like premalignancies. Nat Cell Biol 2014;16:345-356.

29 Yano T, Ferlito M, Aponte A, Kuno A, Miura T, Murphy E, Steenbergen C: Pivotal role of mTORC2 and involvement of ribosomal protein S6 in cardioprotective signaling. Circ Res 2014;114:1268-1280.

-30 Stemke-Hale K, Gonzalez-Angulo AM, Lluch A, Neve RM, Kuo WL, Davies M, Carey M, Hu Z, Guan Y, Sahin A, Symmans WF, Pusztai L, Nolden LK, Horlings H, Berns K, Hung MC, van de Vijver MJ, Valero V, Gray JW, Bernards R, Mills GB, Hennessy BT: An integrative genomic and proteomic analysis of PIK3CA, PTEN, and AKT mutations in breast cancer. Cancer Res 2008;68:6084-6091.

-31 Miller TW, Forbes JT, Shah C, Wyatt SK, Manning HC, Olivares MG, Sanchez V, Dugger TC, de Matos Granja N, Narasanna A, Cook RS, Kennedy JP, Lindsley CW, Arteaga CL: Inhibition of mammalian target of rapamycin is required for optimal antitumor effect of HER2 inhibitors against HER2-overexpressing cancer cells. Clin Cancer Res 2009;15:7266-7276.

-32 Lu CH, Wyszomierski SL, Tseng LM, Sun MH, Lan KH, Neal CL, Mills GB, Hortobagyi GN, Esteva FJ, Yu D: Preclinical testing of clinically applicable strategies for overcoming trastuzumab resistance caused by PTEN deficiency. Clin Cancer Res 2007;13:5883-5888. 